\title{
Multifidus Muscles Lipid Content Is Associated with Intervertebral Disc Degeneration: A Quantitative Magnetic Resonance Imaging Study
}

\author{
Izaya Ogon ${ }^{1}$, Tsuneo Takebayashi ${ }^{2}$, Hiroyuki Takashima ${ }^{1}$, Tomonori Morita ${ }^{1}$, Tsutomu Oshigiri ${ }^{1}$, \\ Yoshinori Terashima ${ }^{1}$, Mitsunori Yoshimoto ${ }^{1}$, Toshihiko Yamashita ${ }^{1}$ \\ ${ }^{1}$ Department of Orthopaedic Surgery, Sapporo Medical University School of Medicine, Sapporo, Japan \\ ${ }^{2}$ Department of Orthopaedic Surgery, Sapporo Maruyama Orthopaedic Hospital, Sapporo, Japan
}

\begin{abstract}
Study Design: Cross-sectional study.
Purpose: To determine the association between fatty degeneration of the multifidus muscle (Mm) and intervertebral disc degeneration (IVDD) using quantitative magnetic resonance imaging (MRI).

Overview of Literature: Few studies have reported on quantitative MRI analysis of the relation between the Mm and IVDD.

Methods: The subjects with chronic low back pain comprised 45 patients (19 males, 26 females; mean age, 63.8 2.0 years; range, 41-79 years). We analyzed the intramyocellular lipids (IMCL) and extramyocellular lipids (EMCL) of the Mm using magnetic resonance spectroscopy. The T2 values of the anterior annulus fibrosus (AF), nucleus pulposus (NP), and posterior AF were evaluated using MRI T2 mapping. We compared the possible correlations of IMCL and EMCL of the Mm with the T2 values of anterior AF, NP, and posterior AF.

Results: There was a significant negative correlation between IMCL and T2 values of the anterior AF $(r=-0.65, p<0.01)$. There were no significant correlations between the IMCL and T2 values of NP $(r=-0.16, p=0.30)$ and posterior $A F(r=0.07, p=0.62)$. There were no significant correlations between the EMCL and T2 values of the anterior AF $(r=-0.11, p=0.46), \mathrm{NP}(r=0.15, p=0.32)$, and posterior AF $(r=0.07, p=0.66)$. After adjustment for age and sex using multiple linear regression analysis, there was a significant negative correlation between the IMCL and T2 values of anterior AF (standardized partial regression coefficient $=-0.65, p<0.01$ ).

Conclusions: The results indicated that IMCL of the Mm might be accompanied with anterior AF degeneration. Therapeutic exercises using IMCL of the Mm as evaluation index might have the potential to identify novel targets for the treatment and prevention of IVDD.
\end{abstract}

Keywords: Paraspinal muscle; Intervertebral disc; Low back pains; Magnetic resonance spectroscopy

\section{Introduction}

Low back pain (LBP) is a commonly observed condition and is one of the most serious physiological concerns worldwide [1]. Trunk muscles are important for normal spinal function and are etiologically significant in LBP [2]. In particular, the multifidus muscle $(\mathrm{Mm})$ - the most medially located back muscle and the largest muscle that

\footnotetext{
Received Sep 30, 2018; Revised Dec 2, 2018; Accepted Dec 23, 2018

Corresponding author: Izaya Ogon

Department of Orthopaedic Surgery, Sapporo Medical University School of Medicine, Japan

291, Soutn-1, West-16, Chuo-ku, Sapporo, 060-8543, Japan

Tel: +81-11-611-2111, Fax: +81-11-641-6026, E-mail: ogon.izaya@sapmed.ac.jp
} 
spans the lumbosacral junction-maintains the erector posture of the trunk as well as abducts and rotates the trunk [3]. Furthermore, intervertebral disc degeneration (IVDD) is considered the principal tissue responsible for LBP [4].

Recently, several studies have performed quantitative evaluation of the $\mathrm{Mm}$ fatty degeneration with magnetic resonance spectroscopy (MRS) [5-10] and IVDD with magnetic resonance imaging (MRI) T2 mapping [11-13]. First, MRS analysis of muscle physiology has facilitated detailed analyses of the muscular fat masses by recording the concentration of intramyocellular lipids (IMCL) and extramyocellular lipids (EMCL) [8-10,14-17]. IMCL cannot be visually detected using conventional MRI because they are stored in the form of spheroid droplets in the cytoplasm of muscle cells in close contact with skeletal mitochondria $[9,10]$ and are directly used as an energy source by the mitochondria. Physical inactivity adversely affects skeletal muscle metabolism, manifesting as poor oxidative capacity $[18,19]$, lower glycogen storage, and decreased capillary density [20]. In contrast, EMCL are defined as more or less compact areas of adipose tissue in the subcutaneous layers or along fasciae and are considered metabolically inactive lipid deposits involved in the reduced functionality associated with obesity and a sedentary lifestyle $[8,14,15]$. Lost muscle strength is associated with EMCL accumulation, which can interfere with sufficient muscle nutrition $[8,15,16]$. In our previous study [5-7], IMCL using the MRS analyses in the Mm of patients with chronic low back pain (CLBP) was significantly higher than that in the control subjects. Next, MRI T2 mapping utilizes the T2 values for the quantification of moisture content and the collagen sequence breakdown of intervertebral disc (IVD). In a previous work [12], we used MRI T2 mapping to quantify the extent of IVDD, which showed a decrease in the $\mathrm{T} 2$ values with increase in the Pfirrmann classification grade [21]. Visual evaluation of the annulus fibrosus (AF) is difficult with ordinary MRI. However, this strategy using T2 values enables us to quantify the degeneration of the AF.

Although fatty degeneration of the Mm as well as IVDD should be a key structural feature of spinal degeneration, few reports exist regarding the MRI analysis of the relation between the Mm and IVDD. The purpose of this cross-sectional quantitative MRI study was to determine the association between the IMCL and EMCL of the Mm and IVDD.

\section{Materials and Methods}

The institutional review board of the Sapporo Medical University approved this study (IRB approval no., 2621074). All the subjects were provided written and verbal explanations of the study and their consent was obtained before study participation.

\section{Participants}

The subjects comprised patients ( $>41$ years and $<79$ years old) who had non-specific CLBP defined as pain, stiffness, and discomfort in the lower back from the 12th rib to the lumbar or the lumbosacral area, wherein the source was difficult to identify, and where the symptoms had persisted despite conservative treatments, such as medication and therapeutic exercise, for $>3$ months. The exclusion criteria included neoplasm, infection, fracture, or history of lumbar vertebral surgery. LBP was assessed using the Visual Analog Scale (VAS, 0-100) after a washout period of minimum 4 weeks. We identified patients whose VAS scores were $>30 \mathrm{~mm}$. The subjects comprised 45 patients (19 males, 26 females; mean age, $63.8 \pm 2.0$ years; range, 41-79 years).

\section{Magnetic resonance spectroscopy}

We used the previously described MRI protocol [5-7]. In brief, the Signa HDx 1.5T MRI system (GE Healthcare, Milwaukee, WI, USA) with a spine coil was used to obtain the T2-weighted sagittal and transverse magnetic resonance (MR) images. Using these images, the proton MRS volume of interest (VOI) was positioned in the center of

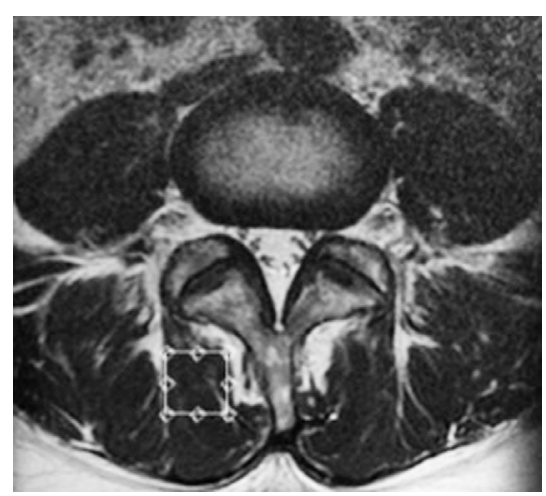

Fig. 1. Volume of interest for magnetic resonance spectroscopy measurements was set in the multifidus muscle on the right side as indicated on the $\mathrm{T} 2$ weighted-image at the $L 4 / 5$ level. 


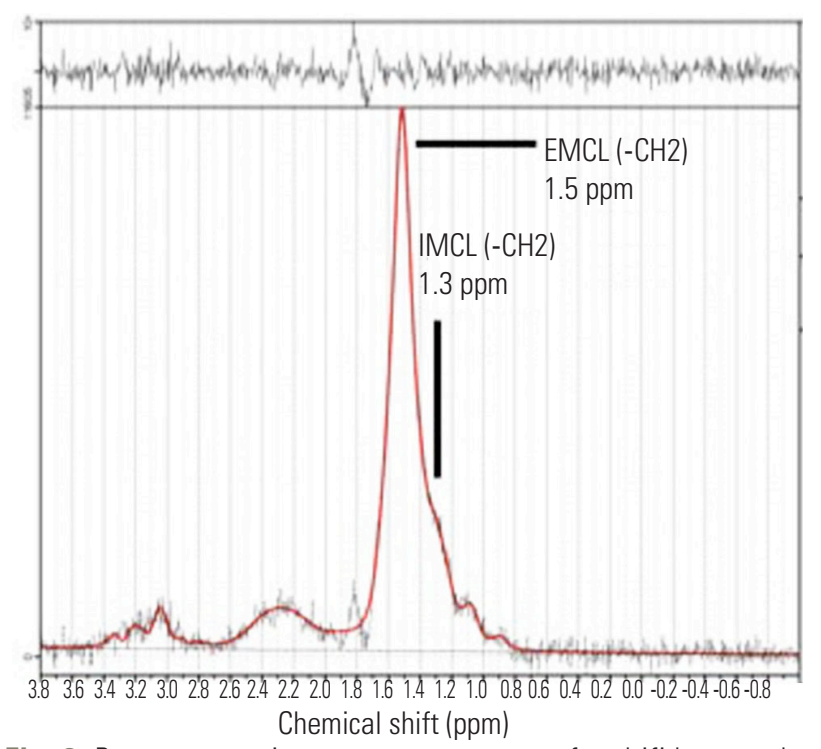

Fig. 2. Proton magnetic resonance spectrum of multifidus muscle analyzed using LCModel software. The following metabolites are identified: IMCLs (-CH2) methylene protons at 1.3 ppm; EMCLs (-CH2) methylene protons at $1.5 \mathrm{ppm}$. IMCLs, intramyocellular lipids; EMCLs, extramyocellular lipids.

the $\mathrm{Mm}$ at L4/5 for the right side (Fig. 1). Single-voxel point-resolved spectroscopy sequence was performed with the following parameters: repetition time (TR), 2,000 $\mathrm{ms}$; echo time (TE), $35 \mathrm{~ms}$; average number of signals, 64; VOI size, $15 \times 15 \times 15 \mathrm{~mm}(3.4 \mathrm{~mL})$; and acquisition time, 164 seconds. Analyses of MR spectroscopic data were also performed using a previously described method [57]. The spectral data obtained were used to measure the IMCL and EMCL using the LCModel software (Stephen Provencher Inc., Oakville, ON, Canada). Data were transferred from the scanners to a Linux workstation, and metabolite quantification was performed with eddy current correction and water scaling. Data for the IMCL (1.3 ppm) and EMCL (1.5 ppm) corresponding to the methylene protons were used for statistical analyses. The assessments of the IMCL and EMCL were automatically scaled to an unsuppressed water peak (4.7 ppm) and expressed in institutional units. These data are graphically displayed with the chemical shift along the $\mathrm{x}$-axis, allowing the identification of the metabolites. Peak intensity has been plotted on the y-axis (Fig. 2). We excluded the subjects with $\mathrm{Mm}>15 \%$ of the \%standard deviation of EMCL or IMCL on the LCModel.

\section{Magnetic resonance imaging $\mathrm{T} 2$ mapping}

We used the MRI protocol and methods of analysis for
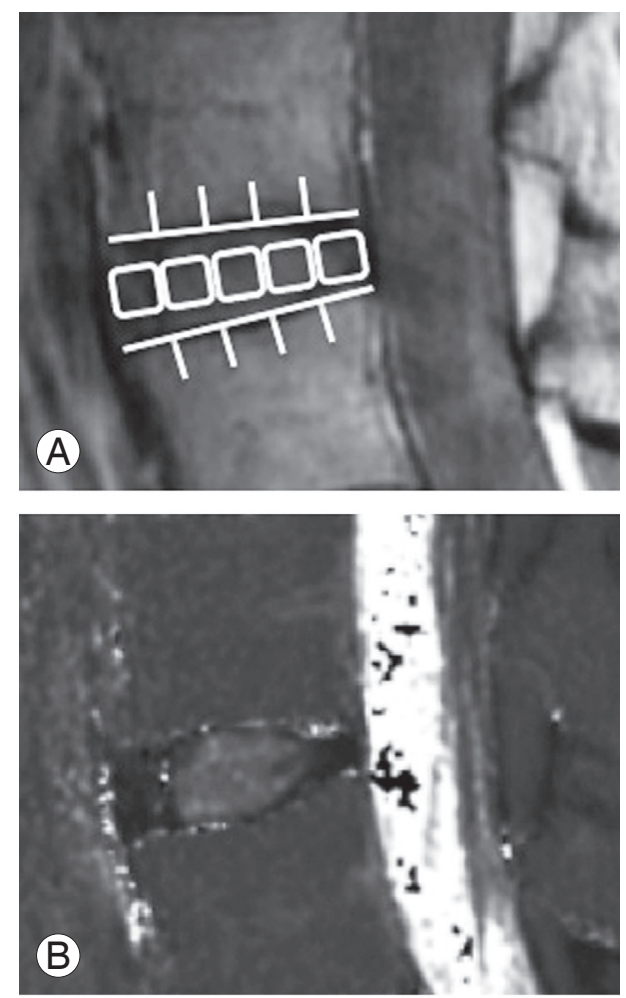

Fig. 3. In the second echo image, the disc was divided into five areas, designating the front fifth of the anterior $A F$, the middle fifth of the nucleus pulposus, and the last fifth of the posterior $A F(A)$. In the same region, we measured the mean values $(B)$. $A F$, annulus fibrosus.

MRI T2 mapping that have been previously described [11-13]. Briefly, a T2 map was created at L4/5 level using the $\mathrm{T} 2$ values in the midsagittal portion of the sagittal sections centered on the lumbar midline region with optimized 8 echo multi-spin echo (TR/first echo TE, last echo TE, 1,000/14.8, 118.6; receiver bandwidth, $\pm 15.63 \mathrm{kHz}$; field of view, $22 \mathrm{~cm}$; matrix, $320 \times 256$; slice thickness/gap, $4 \mathrm{~mm} / 4 \mathrm{~mm}$; 5 slices; number of excitations, 2; total scan time, 8 minutes and 34 seconds) obtained with an Advantage Workstation (version 4.4, Functool; GE Healthcare). However, the first echo from the multi-spin system was excluded to minimize the effect of the stimulated echo. The T2 map was calculated in each pixel from the signal intensity (SI) in the respective TE using the following calculating formula: SI 1/4 e_TE=T2.

For measurement, the disc was divided into five equal areas, designating the front fifth of the anterior AF, the middle fifth of the nucleus pulposus (NP), and the last fifth of the posterior AF, as per previous reports [11-13]. The mean values in the region of interest were measured (Fig. 3). T2 values were measured by a researcher with 

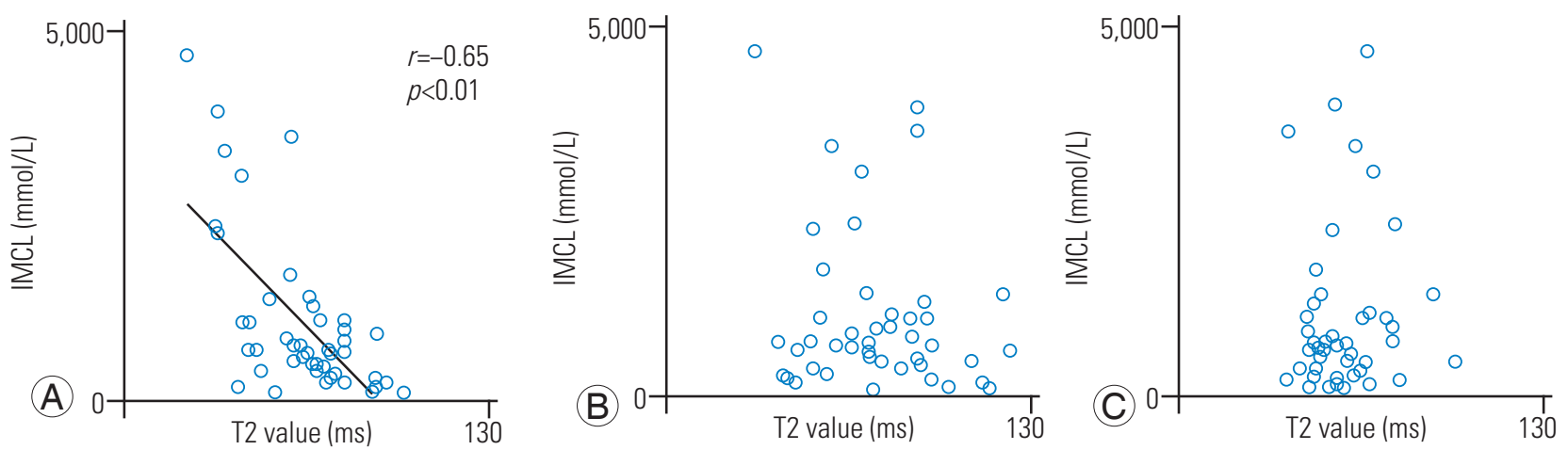

Fig. 4. Relationships of the IMCLs and of the multifidus muscle with the T2 values of the anterior AF, NP, and posterior AF. The correlation coefficient between IMCL and anterior AF (A) indicated significant negative correlation. The correlation coefficient between IMCL and T2 values of NP (B) and posterior $\mathrm{AF}(\mathrm{C})$ indicated a non-significant correlation. IMCL, intramyocellular lipid; $\mathrm{AF}$, annulus fibrosus; NP, nucleus pulposus.
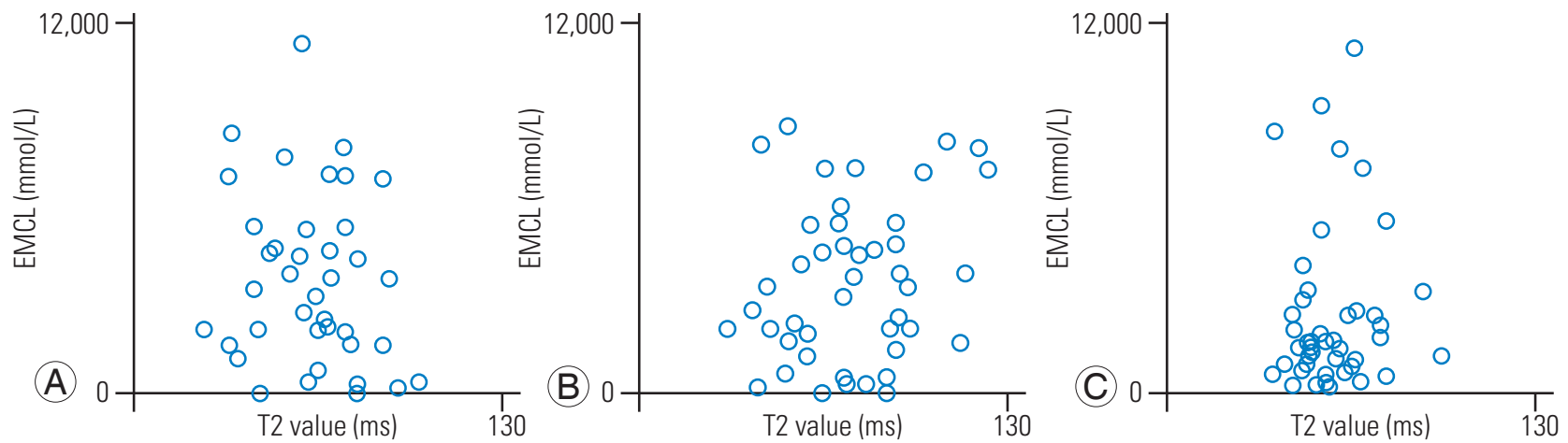

Fig. 5. Relationships of EMCLs and of the Mm with the T2 values of anterior AF, NP, and posterior AF. The correlation coefficient between the EMCL and $\mathrm{T} 2$ values of anterior $\mathrm{AF}(\mathrm{A}), \mathrm{NP}(\mathrm{B})$, and posterior $\mathrm{AF}(\mathrm{C})$ indicated a non-significant correlation. EXCL, extramyocellular lipid; $A F$, annulus fibrosus; NP, nucleus pulposus.

$\mathrm{PhD}$ (H.T. who had 12 years of experience in spine MR image analysis) using MedCalc (ver. 10.2.0.0; MedCalc Software, Mariakerke, Belgium).

\section{Statistical analyses}

We compared the possible correlations of IMCL and EMCL of the Mm with the T2 values of the anterior AF, NP, and posterior AF using Pearson's correlation coefficient test. We performed multiple linear regression analysis to eliminate the influence of age and sex. A $p<0.05$ indicated statistical significance. All the numerical data are expressed as mean \pm standard error of the mean values.

\section{Results}

The mean body mass index was $24.6 \pm 0.5 \mathrm{~kg} / \mathrm{m}^{2}$. The mean $\mathrm{T} 2$ values in IVD were $63.4 \pm 2.7 \mathrm{~ms}$ for the anterior $\mathrm{AF}$, $73.5 \pm 3.3 \mathrm{~ms}$ for NP, and $58.0 \pm 1.9 \mathrm{~ms}$ for the posterior AF.
The mean IMCL of the Mm was $10.6 \pm 1.62\left(\times 10^{2}\right) \mathrm{mmol} / \mathrm{L}$, and the mean EMCL was $3.72 \pm 0.41\left(\times 10^{3}\right) \mathrm{mmol} / \mathrm{L}$. There was a significant negative correlation between the IMCL and $\mathrm{T} 2$ values of anterior AF $(r=-0.65, p<0.01)$ (Fig. 4A). There were no significant correlations between IMCL and T2 values of NP ( $r=-0.16, p=0.30)$ (Fig. $4 \mathrm{~B}$ ) and posterior AF ( $r=0.07, p=0.62)$ (Fig. 4 C). There were no significant correlations between the EMCL and $\mathrm{T} 2$ values of anterior $\mathrm{AF}(r=-0.11, p=0.46)$ (Fig. 5A), NP $(r=0.15, p=0.32)$ (Fig. $5 \mathrm{~B})$, and posterior AF ( $r=0.07, p=0.66)$ (Fig. $5 \mathrm{C}$ ). Table 1 shows multiple linear regression analysis adjusted for age and sex. There was a significant negative correlation between the IMCL and T2 values of anterior AF (standardized partial regression coefficient $=-0.65, p<0.01$ ).

\section{Discussion}

The Mm and IVD are important for the proper functioning of the lumbar spinal column and degeneration in 
Table 1. Multiple linear regression analysis adjusted for age and sex

\begin{tabular}{|c|c|c|c|c|c|}
\hline Dependent variable & Independent variable & Regression coefficient & Standard error & $\begin{array}{l}\text { Standardized partial } \\
\text { regression coefficient }\end{array}$ & $p$-value \\
\hline \multirow[t]{3}{*}{ Intramyocellular lipids } & Anterior AF & -39.11 & 6.90 & -0.65 & $<0.01$ \\
\hline & NP & -6.43 & 7.53 & -0.13 & 0.40 \\
\hline & Posterior AF & 5.10 & 13.15 & 0.06 & 0.70 \\
\hline \multirow[t]{3}{*}{ Extramyocellular lipids } & Anterior AF & -16.56 & 23.19 & -0.11 & 0.48 \\
\hline & NP & 21.72 & 18.92 & 0.18 & 0.26 \\
\hline & Posterior AF & 10.90 & 33.30 & 0.05 & 0.75 \\
\hline
\end{tabular}

AF, annulus fibrosus; NP, nucleus pulposus.

either impairs function [22,23]. Few MRI studies have examined the association between paraspinal muscle fatty degeneration and IVDD. Kader et al. [22] showed a tendency of $\mathrm{Mm}$ fatty degeneration being associated with the number of degenerated discs. Teichtahl et al. [23] revealed that severe disc degeneration at all intervertebral levels was associated with high fat content of the paraspinal muscles. Sun et al. [24] reported that Mm atrophy and IVDD were positively correlated at the L3/L4 disc level. However, these studies used visual evaluation to evaluate muscular fatty degeneration and IVDD.

In this study, we used quantitative MRI evaluation methods, such as MRS and MRI T2 mapping, and demonstrated a correlation between IMCL of the Mm and T2 values of anterior AF. Previously, we have identified the associations of spino-pelvic alignment (lumbar lordosis and sagittal vertical axis) with the IMCL of the Mm [5]. The Mm provides two-thirds of the spinal segmental stability [25] and is important for maintaining spinal alignment [26]. In addition, we evaluated the extent of IVDD and compared this with the T2 values in degenerative spondylolisthesis (DS) and no-spondylolisthesis groups; we found that the $\mathrm{T} 2$ values decreased in the anterior AF of IVD in the DS group [13]. It is speculated that dysfunction of the Mm leads to anterior gliding because of reduced resistance to anterior shear forces. More mechanical stress loaded at the anterior part of the IVD might lead to pressure on the anterior AF. Thus, the $\mathrm{Mm}$ as well as anterior AF of IVD are important for proper functioning of the lumbar spinal column and are cross-correlated to each other.
IVDD [27] and collapsed vertebral bodies [28] irreversibly develop with advancing age; however, an increase in the IMCL of the Mm can probably improve if conservative treatments, such as therapeutic exercise, are initiated. There is a correlation between IMCL of the Mm and the T2 values of anterior AF; therefore, it is expected that therapeutic exercises might have the potential to identify novel targets for the treatment and prevention of IVDD.

This study has certain limitations. First, this study has a cross-sectional design; thus, it cannot be determined whether associations between IMCL increase of the Mm and IVDD are a cause or the result of one another. Longitudinal studies will help address such concerns. Second, we did not perform any evaluation about other factors involved in IVDD, such as smoking, diabetes mellitus, hypertension, and physical activity.

\section{Conclusions}

The results indicated that the IMCL of the Mm might be accompanied with posterior AF degeneration. Therapeutic exercises using IMCL of the Mm as evaluation index might have the potential to identify novel targets for the treatment and prevention of IVDD.

\section{Conflict of Interest}

No potential conflict of interest relevant to this article was reported. 


\section{Author Contributions}

Conception and design: IO, TT, HT; acquisition of data: IO, HT; analysis and interpretation of data: IO, HT; drafting the article: IO, TT; critically revising the article: all authors; reviewed submitted version of manuscript: all authors; approved the final version of the manuscript on behalf of all authors: TT; statistical analysis: IO; administrative/technical/material support: TM, TO, YT, MY, TY; and study supervision: TY.

\section{References}

1. Deyo RA, Weinstein JN. Low back pain. N Engl J Med 2001;344:363-70.

2. Cholewicki J, Panjabi MM, Khachatryan A. Stabilizing function of trunk flexor-extensor muscles around a neutral spine posture. Spine (Phila Pa 1976) 1997;22:2207-12.

3. Yoshihara K, Shirai Y, Nakayama Y, Uesaka S. Histochemical changes in the multifidus muscle in patients with lumbar intervertebral disc herniation. Spine (Phila Pa 1976) 2001;26:622-6.

4. Hurri H, Karppinen J. Discogenic pain. Pain 2004;112:225-8.

5. Ogon I, Takebayashi T, Takashima H, et al. Magnetic resonance spectroscopic analysis of multifidus muscles lipid content and association with spinopelvic malalignment in chronic low back pain. Br J Radiol 2017;90:20160753.

6. Takashima H, Takebayashi T, Ogon I, et al. Evaluation of intramyocellular and extramyocellular lipids in the paraspinal muscle in patients with chronic low back pain using MR spectroscopy: preliminary results. Br J Radiol 2016;89:20160136. https://doi. org/10.1259/bjr.20160136.

7. Takashima H, Takebayashi T, Ogon I, et al. Analysis of intra and extramyocellular lipids in the multifidus muscle in patients with chronic low back pain using MR spectroscopy. Br J Radiol 2018;91:20170536.

8. Boesch C, Machann J, Vermathen P, Schick F. Role of proton MR for the study of muscle lipid metabolism. NMR Biomed 2006;19:968-88.

9. Boesch C. Musculoskeletal spectroscopy. J Magn Reson Imaging 2007;25:321-38.

10. Boesch C, Kreis R. Observation of intramyocellular lipids by $1 \mathrm{H}$-magnetic resonance spectroscopy. Ann
N Y Acad Sci 2000;904:25-31.

11. Ogon I, Takebayashi T, Takashima H, et al. Analysis of chronic low back pain with magnetic resonance imaging T2 mapping of lumbar intervertebral disc. J Orthop Sci 2015;20:295-301.

12. Takashima H, Takebayashi T, Yoshimoto M, et al. Correlation between $\mathrm{T} 2$ relaxation time and intervertebral disk degeneration. Skeletal Radiol 2012;41:1637.

13. Takashima H, Takebayashi T, Yoshimoto M, et al. Investigation of intervertebral disc and facet joint in lumbar spondylolisthesis using T2 mapping. Magn Reson Med Sci 2014;13:261-6.

14. Srikanthan P, Singhal A, Lee CC, et al. Characterization of intra-myocellular lipids using $2 \mathrm{~d}$ localized correlated spectroscopy and abdominal fat using MRI in type 2 diabetes. Magn Reson Insights 2012;5:2936.

15. Velan SS, Said N, Durst C, et al. Distinct patterns of fat metabolism in skeletal muscle of normal-weight, overweight, and obese humans. Am J Physiol Regul Integr Comp Physiol 2008;295:R1060-5.

16. Jucker BM, Schaeffer TR, Haimbach RE, et al. Reduction of intramyocellular lipid following short-term rosiglitazone treatment in Zucker fatty rats: an in vivo nuclear magnetic resonance study. Metabolism 2003;52:218-25.

17. Schrauwen-Hinderling VB, Hesselink MK, Schrauwen P, Kooi ME. Intramyocellular lipid content in human skeletal muscle. Obesity (Silver Spring) 2006;14:357-67.

18. Dube JJ, Amati F, Stefanovic-Racic M, Toledo FG, Sauers SE, Goodpaster BH. Exercise-induced alterations in intramyocellular lipids and insulin resistance: the athlete's paradox revisited. Am J Physiol Endocrinol Metab 2008;294:E882-8.

19. Konopka AR, Suer MK, Wolff CA, Harber MP. Markers of human skeletal muscle mitochondrial biogenesis and quality control: effects of age and aerobic exercise training. J Gerontol A Biol Sci Med Sci 2014;69:371-8.

20. Zoladz JA, Semik D, Zawadowska B, et al. Capillary density and capillary-to-fibre ratio in vastus lateralis muscle of untrained and trained men. Folia Histochem Cytobiol 2005;43:11-7.

21. Pfirrmann CW, Metzdorf A, Zanetti M, Hodler J, Boos N. Magnetic resonance classification of lumbar 
intervertebral disc degeneration. Spine (Phila Pa 1976) 2001;26:1873-8.

22. Kader DF, Wardlaw D, Smith FW. Correlation between the MRI changes in the lumbar multifidus muscles and leg pain. Clin Radiol 2000;55:145-9.

23. Teichtahl AJ, Urquhart DM, Wang Y, et al. Modic changes in the lumbar spine and their association with body composition, fat distribution and intervertebral disc height: a 3.0 T-MRI study. BMC Musculoskelet Disord 2016;17:92.

24. Sun D, Liu P, Cheng J, Ma Z, Liu J, Qin T. Correlation between intervertebral disc degeneration, paraspinal muscle atrophy, and lumbar facet joints degeneration in patientswith lumbar disc herniation. BMC Musculoskelet Disord 2017;18:167.
25. Wilke HJ, Wolf S, Claes LE, Arand M, Wiesend A. Stability increase of the lumbar spine with different muscle groups: a biomechanical in vitro study. Spine (Phila Pa 1976) 1995;20:192-8.

26. Takemitsu Y, Harada Y, Iwahara T, Miyamoto M, Miyatake Y. Lumbar degenerative kyphosis: clinical, radiological and epidemiological studies. Spine (Phila Pa 1976) 1988;13:1317-26.

27. Haefeli M, Kalberer F, Saegesser D, Nerlich AG, Boos N, Paesold G. The course of macroscopic degeneration in the human lumbar intervertebral disc. Spine (Phila Pa 1976) 2006;31:1522-31.

28. Chrischilles EA, Butler CD, Davis CS, Wallace RB. A model of lifetime osteoporosis impact. Arch Intern Med 1991;151:2026-32. 